\title{
Burr Detection by Using Vision Image
}

\author{
Kuang-Chyi Lee, Han-Pang Huang and Shui-Shong Lu \\ Robotics Laboratory. Department of Mechanical Engineering, National Taiwan University, Taipei, Taiwan, ROC
}

In this paper, a practical force model for the deburring process is first presented. It will be shown that the force model is more general than Kazerooni's model and it is suitable for both upcut and down-cut grinding. In terms of this force model, an algorithm of burr detection by using a $2 D$ vision image is proposed. In the burr detection algorithm, the relevant data of burrs, such as frequency, cross-section area, and height are simplified so that they are functions of the burr contour only. Then, a fast tracking method of the burr contour (BCTM) is developed to obtain the contour data. Experiments show that the BCTM of this passive (i.e. without lighting) image system can be as fast as $18.2 \mathrm{~Hz}$ and its precision is $0.02 \mathrm{~mm}$, so online burr detection and control by using the vision sensor is feasible.

Keywords: Deburring; Force model; Vision; Grinding

\section{Introduction}

Most machining processes can produce raised edges or burrs on machined surfaces. Burrs not only affect the appearance and performance of products but also can cause inconvenience during machining; thus, deburring is imperative in a machining process. Recently, robots have been used in the deburring process to improve the efficiency of machining and the quality of products. In other words, robotic deburring may become a key technology to provide an advanced manufacturing system for high quality.

In the robotic deburring process, it is necessary to feedback to the robot the data of the force, the position, and the burr. Owing to the use of rotary files and grinders in the robotic deburring process, the deburring process is an analogy to a grinding process. Although Rubenstein [1] proposed the mechanics of grinding, the model is too complicated to

Accepted for publication: 7 January 1992

Correspondence and offprint requests to: Han-Pang Huang, Robotics Laboratory, Department of Mechanical Engineering, National Taiwan University, No 1 Roosevelt Road, Sec. 4, Taipei, Taiwan 10764, Republic of China. implement. In order to facilitate the controller design and the burr measurement in the process, it is desired to develop a feasible force model for the up-cut and down-cut grinding processes. Also, the data of burrs should be carefully modified from 2D image information.

Owing to the irregularity and the large variation of size, it is difficult to measure burrs. If touch sensors are used to measure the data of burrs, the measurement is easily disturbed by surrounding burrs. Similarly, if an active vision system, in which the structured light of laser beams or other light sources with longer wavelengths are used, is adopted to measure the burrs, the phenomena of interference and diffraction easily occur. The passive vision system used in this paper neither contacts the burr nor causes the phenomenon of interference.

First, this paper proposes a force model of the deburring process. The model is based on the linear function of the cross-section area of the burr and the height of the burr. Next, two types of burr models are constructed for the 2D image system. The frequency, the cross-section area, and the height of the burr will be functions of the burr contour. Then a burr detection algorithm using the passive $2 \mathrm{D}$ image is proposed. In the algorithm, a fast tracking method of the burr contour (BCTM) is developed to obtain the contour data. Finally, an experiment is performed to justify the proposed algorithm. The experiment indicates that the speed of the BCTM can be up to $18.2 \mathrm{~Hz}$ with a measurement precision of $0.02 \mathrm{~mm}$.

\section{Mechanics of Deburring Processes}

Rubenstein [1] divided the cutting force in grinding into the chip formation force, the force component arising from the finite radius of curvature of the cutting edge, the friction force between the flank wear land and the workpiece, the force for grains to cut the workpiece, the force for grains to plough the workpiece, and the friction force between the wheel bond and the workpiece material. In the actual grinding case, the classification of the above-mentioned cutting forces is quite complicated and each of the classification items is difficult to obtain. During the deburring process, the ploughing force is so small that it can be neglected. In general, the cutting force can be simply represented by the friction force 
and the chip formation force [2]. When there is no chip formation, the grinding process becomes a pure friction process owing to the small normal force. Alternatively, the grinding process becomes a pure chip formation process at which point there is either no friction force or the friction force is much smaller than the chip formation force. Hence, the force model for deburring processes can be obtained as (see Appendix)

$$
\begin{aligned}
& F_{\mathrm{n}}=\frac{2 K_{\mathrm{c}}}{D}\left(\frac{V_{\mathrm{w}}}{V_{\mathrm{s}}}\right) A_{\text {work }}+2 K_{\mathrm{f}} a_{\text {root }} L \\
& F_{\mathrm{t}}=\frac{2 \phi K_{\mathrm{c}}}{D}\left(\frac{V_{\mathrm{w}}}{V_{\mathrm{s}}}\right) A_{\text {work }}+2 \mu K_{\mathrm{f}} a_{\text {root }} L
\end{aligned}
$$

Equations (1) and (2) denote the force model of the grinding process. This force model has several features:

1. It is more general than Kazerooni's force model [2-6]; i.e. Kazerooni's force model is a special case of equations (1) and (2). Note that Kazerooni's force model is

$$
\begin{aligned}
& Z_{\mathrm{w}}=\wedge_{\mathrm{w}}\left(F_{\mathrm{n}(\mathrm{K})}-F_{0}\right) \\
& F_{\mathrm{t}(\mathrm{K})}=\mu F_{\mathrm{n}(\mathrm{K})} \\
& Z_{\mathrm{w}}=V_{\mathrm{w}} A_{\text {work }} \\
& \wedge_{\mathrm{w}}=K_{2} V_{\mathrm{s}}
\end{aligned}
$$

where $F_{0}$ is the threshold thrust force; $F_{\mathrm{n}(\mathrm{K})}$ is the normal grinding force of Kazerooni's model; $F_{\mathrm{t}(\mathrm{K})}$ is the tangential grinding force of Kazerooni's model; $K_{2}$ is the specific metal-removal parameter per wheel speed; $Z_{w}$ is the metalremoval rate; and $\wedge_{w}$ is the metal-removal parameter. Thus,

$$
\begin{aligned}
F_{\mathrm{n}(\mathrm{K})} & =\frac{1}{K_{2}}\left(\frac{V_{\mathrm{w}}}{V_{\mathrm{s}}}\right) A_{\text {work }}+F_{0} \\
F_{\mathrm{r}(\mathrm{K})} & =\phi F_{\mathrm{n}(\mathrm{K})}
\end{aligned}
$$

By comparing equations (4) and (5) with equations (1) and (2), it can be seen that Kazerooni's force model is a special case of equations (1) and (2) with $\mu=\phi$ and $F_{0}$ $=$ constant .

2. The values of $K_{\mathrm{c}}, C_{\mathrm{f}}, \mu, \phi$ in equations (1) and (2) can be evaluated by experiment.

3. Equations (1) and (2) can be applied to both up-cut grinding and down-cut grinding (see Appendix). However, the values of $F_{\mathrm{y}}$ and $F_{\mathrm{h}}$ vary for different cutting cases.

\section{Burr Models}

The force model in equations (1) and (2) describes the characteristics of the deburring surface. The characteristics of burrs are related to the force model through $A_{\text {work }}$ and $a_{\text {root }}$. In practice, a limitation on the range of the tool operating frequency should be imposed [7-10]; i.e. $f_{\mathrm{r}}<f_{\text {tool }}<f_{\text {burr }}$, where $f_{\text {burr }}$ is the frequency of the burr, $f_{r}$ is the first resonant frequency of the robot, and $f_{\text {tool }}$ is the resonant frequency of the end-effector at the normal direction. In order to accurately control the deburring force during the deburring process, the information of burrs should be feedback. Namely $A_{\text {work }}, a_{\text {root }}$

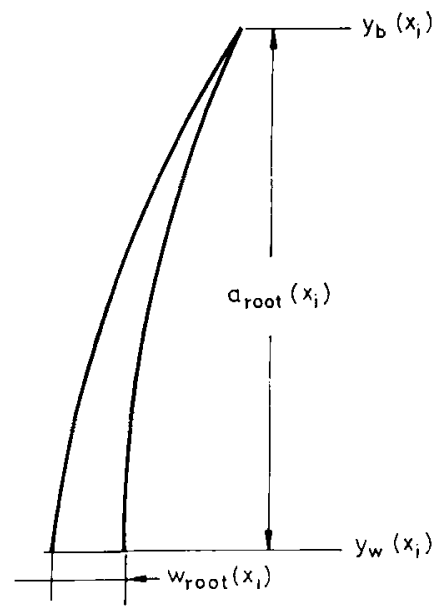

Fig. 1. The configuration of the parabolic burr.

and $f_{\text {burr }}$ need to be detected. But $f_{\text {burr }}$ can be calculated from $A_{\text {burr }}\left(x_{i}\right)$ and $A_{\text {work }}=A_{\text {burr }}+A_{\text {chamfer }}$, where $A_{\text {burr }}$ is the cross-section area of the burr and $A_{\text {chamfer }}$ is the cross-section area of the chamfer. Thus, only $A_{\text {bur }}$ and $a_{\text {root need be }}$ detected. Since only the $2 \mathrm{D}$ image is used, for the sake of fast computation, $A_{\text {bur }}$ will not be detected directly. Therefore, it is desired to develop suitable burr models for the calculation of $A_{\text {burr }}$ and $a_{\text {root }}$. Two types of burr models will be considered, i.e. the parabolic burr and the circular burr.

Consider the case of the parabolic burr. From the geometric relation in Fig. 1, the thickness of the root of the burr, $w_{\text {root }}$ is

$$
w_{\text {root }}\left(x_{i}\right)=k a_{\text {roor }}\left(x_{i}\right)
$$

Thus, we obtain

$$
A_{\text {burr }}\left(x_{i}\right)=\frac{2}{3} a_{\text {ruot }}\left(x_{i}\right) w_{\text {root }}\left(x_{i}\right)=\frac{2}{3} k a_{\text {root }}^{2}\left(x_{i}\right)
$$

where $k$ is a constant for the parabolic burr. The parabolic burr model is suitable for most machining cases.

Next, the case of the circular burr is considered. From the geometric relation in Fig. 2, the radius of the burr, $r$, is

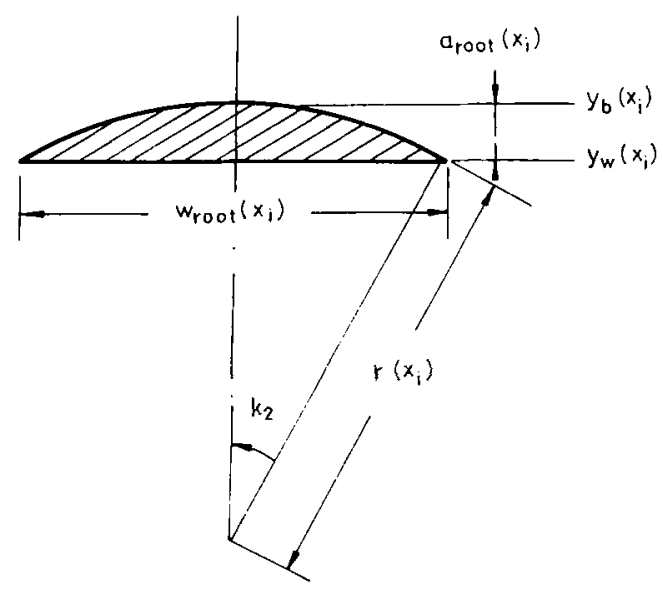

Fig. 2. The configuration of the circular burr. 


$$
r\left(x_{i}\right)=k_{1} a_{\text {roor }}\left(x_{i}\right)
$$

Then, the angle of the burr $\left(k_{2}\right)$ and the root of the burr $\left(w_{\text {root }}\right)$ are

$$
\begin{aligned}
& k_{2}=\cos ^{-1}\left[\left(r\left(x_{i}\right)-a_{\text {root }}\left(x_{i}\right)\right) / r\left(x_{i}\right)\right]=\cos ^{-1}\left[\left(k_{1}-1\right) / k_{1}\right] \\
& w_{\text {root }}\left(x_{i}\right)=2 r\left(x_{i}\right) \sin k_{2}=2 k_{1} \sin k_{2} a_{\text {root }}\left(x_{i}\right)=k_{3} a_{\text {root }}\left(x_{i}\right)
\end{aligned}
$$

Hence, we obtain

$$
\begin{aligned}
A_{\text {burr }}\left(x_{i}\right) & =1 / 2 r^{2}\left(x_{i}\right) k_{2}-1 / 2 w_{\text {root }}\left(x_{i}\right)\left[r\left(x_{i}\right)-a_{\text {root }}\left(x_{i}\right)\right] \\
& =1 / 2\left[k_{1}^{2} k_{2}-k_{3}\left(k_{1}-1\right)\right] a_{\text {root }}^{2}\left(x_{i}\right)
\end{aligned}
$$

Namely,

$$
A_{\text {burr }}\left(x_{i}\right)=1 / 2 k_{4} a_{\text {root }}^{2}\left(x_{i}\right)
$$

Note that $k_{1}, k_{2}, k_{3}, k_{4}$ are constants for the circular burr. The circular burr model can be used to cast the welding bead.

From the above developments, it is clear that $A_{\text {burr }}\left(x_{i}\right)$ is a function of $a_{\text {root }}\left(x_{i}\right)$ only. Hence, the burr height $a_{\text {root }}\left(x_{i}\right)$ is the only data that needs to be detected. However,

$$
a_{\text {root }}\left(x_{i}\right)=y_{\mathrm{b}}\left(x_{i}\right)-y_{\mathrm{w}}\left(x_{i}\right)
$$

where $y_{\mathrm{b}}\left(x_{i}\right)$ is the contour of the burr detected by the 2D image system and $y_{w}\left(x_{i}\right)$ is the contour of the workpiece. The contour of the workpiece $y_{\mathrm{w}}\left(x_{i}\right)$ can be computed from $y_{\mathrm{b}}\left(x_{i}\right)$ by the least-square method or can be input in advance. Thus, $f_{\text {burr }}, w_{\text {root }}\left(x_{i}\right)$, and $A_{\text {burr }}\left(x_{i}\right)$ can be obtained from $a_{\text {root }}\left(x_{i}\right)$, and $a_{\text {root }}\left(x_{i}\right)$ can be computed from $y_{\mathrm{b}}\left(x_{i}\right)$. In other words, $y_{\mathrm{b}}\left(x_{i}\right)$ is the only data needed to be acquired by the 2D passive vision system.

\section{Algorithm for Burr Detection}

The passive vision system neither contacts the burr nor causes the phenomenon of interference. In addition, the processing of a $2 \mathrm{D}$ image is much faster than that of a $3 \mathrm{D}$ image. Therefore, a passive system with a 2D image is used to detect the burr's information. The block diagram of the burr detection system is shown in Fig. 3. The functions of the block diagram are described as follows.

\subsection{Hardware of the Image System}

The 2D image is grabbed by the IM- 1280 system developed by Matrox Inc. The IM-1280 system consists of a RTP (Real Time Processor) board with a $4 \mathrm{MB}$ frame buffer, an IMASD board for the RS170 B/W input video frame grabber, and a baseboard for display and system controllers. These cards are plugged into the slots of a PC-AT. In addition, a PULNIX B/W CCD camera with a magnification lens is used.

\subsection{Thresholding Methods of the Image System}

There are many thresholding methods for taking the binary image [11-18]. This paper uses a maximum histogram valley method to find the proper threshold value [19]. In the maximum histogram valley method, the histogram of a picture

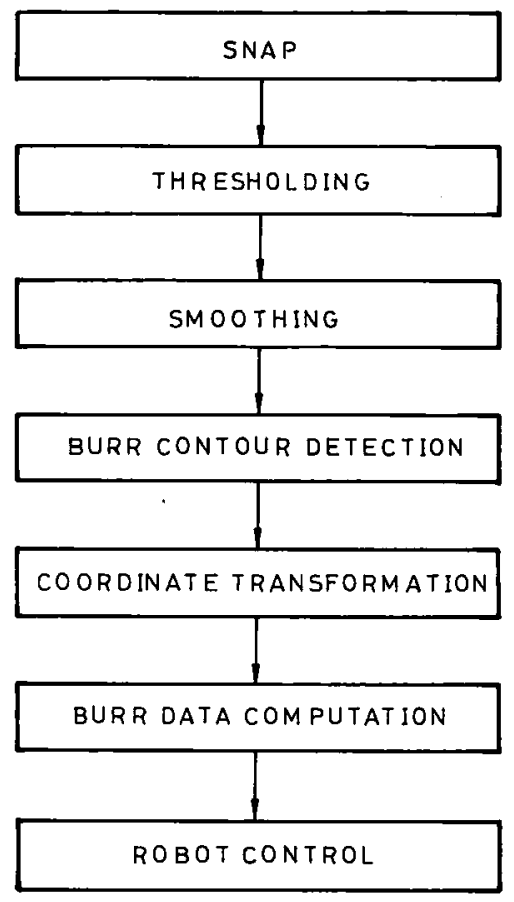

Fig. 3. The block diagram of burr detection by using the $2 \mathrm{D}$ vision image.

is first obtained. Next, each valley in the histogram is found by eight grey levels. Then, the quantities between any two adjacent valleys are summed up. Finally, the grey level of the middle valley between the first two largest sums is chosen as the required threshold value. This method is performed only for the first image picture to acquire the proper threshold value; thereafter, this threshold is used for all later pictures. The thresholding procedure of the maximum histogram valley method is very fast and was quite successful in our experiments.

\subsection{Smoothing Methods of the Image System}

The detection of the burr is greatly affected by the pepperand-salt noise in the image $[13,20]$. This paper adopts two ways to remove the pepper-and-salt noise, viz. the morphology method and the length method. In the morphology method, the binary image is processed several times by erosion and dilation operations with respect to 4-connected neighbours. First, a $3 \times 3$ convolution of the local minimum is performed three times on the image followed by a $3 \times 3$ convolution of the local maximum three times. Next, a $3 \times 3$ convolution of the local maximum is performed three times on the image, which is again followed by a $3 \times 3$ convolution of the local minimum three times. Although the morphology method takes $\frac{12}{30} \mathrm{~s}$, it is required only for the first image picture. In the length method, the burr contour is obtained through the burr detection algorithm. If the length of the detected burr is too small, it is ignored and the address of $P$ (refer to the burr detection algorithm) is reset to the first point of the burr, then the burr detection algorithm is resumed. 


\subsection{Algorithm for Burr Detection}

From the development of burr models, $A_{\text {burr }}\left(x_{i}\right)$ and $f_{\text {burr }}$ depend only on $a_{\text {root }}\left(x_{i}\right)$ and $a_{\text {root }}\left(x_{i}\right)=y_{\mathrm{b}}\left(x_{i}\right)-y_{\mathrm{w}}\left(x_{i}\right)$. Furthermore, the contour of the workpiece $y_{w}\left(x_{i}\right)$ is known a priori or it can be computed from $y_{\mathrm{b}}\left(x_{i}\right)$ by the least-squares method. Thus, the complete burr data can be computed from the burr contour $y_{\mathrm{b}}\left(x_{i}\right)$ as long as it is known.

For detecting the contour of the burr, the contourfollowing method given in [19], which is only suitable for the closed contour and uni-direction (either clockwise or counterclockwise), must be modified to adapt to the situation of open contour and mixed directions. Therefore, a burr contour tracking method (BCTM) is proposed. Consider the range of the window from $\left(x_{1}, y_{1}\right)$ to $\left(x_{2}, y_{2}\right)$ on the binary image, as shown in Fig. 4. $E$ and $O$ in the figure represent the grey levels of the environment and the object, respectively. A $3 \times 3$ mask, as given in Fig. 5, will be used in the BCTM. In Fig. 5, $P$ represents the address of the point concerned and $U, D, R$, and $L$ represent the addresses of the neighbours of $P$. The 4-connected neighbourhood processing method will be used in the algorithm. Thus, the BCTM algorithm can be described in terms of C-language format as follows

1. $E=*\left(x_{1}, y_{1}\right) ; P=\left(x_{1}, y_{1}\right)$;

2. while $\left((* P==E) \& \&\left(P(y) !=y_{2}\right)\right)\left\{P=D_{i}\right\}$

3. while $\left((* P==E) \& \&\left(P(x) !=x_{2}\right)\right)\{P(x)=P(x)+1$; $P(y)=y_{1} ;$ goto $\left.2 ;\right\}$

4. while $(* U !=E)\{P=U ;\}$

5. record $P$;

6. while $((* U==E) \& \&(* D==E))\{* P=E$; delete $P$; $P=L ;\}$

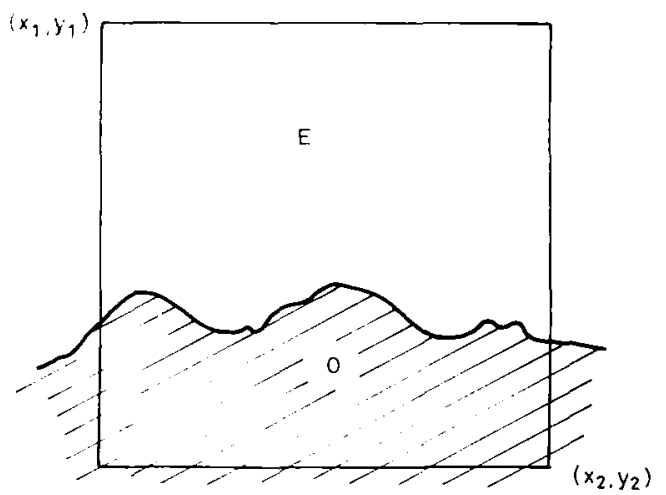

Fig. 4. The binary image of the burr and its environment for the BCTM (Burr Contour Tracking Method).

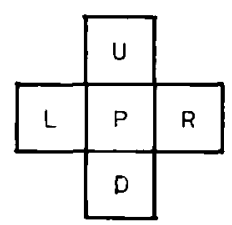

Fig. 5. The mask for the BCTM.
7. if(*R! = E) $\{P=R$; while $(* U !=E)\{P=U ;\}\}$ else $\{P=R ;$ while $(* D==E)\{P=D ;\}\}$

8. record $P$; if $\left(P(x) !=x_{2}\right)$ goto 6; else stop;

Note that $* P$ denotes the grey level of the address $P ; P(x)$ is the $x$-address of $P$; and $P(y)$ is the $y$-address of $P$. In step 1 , the grey level of the environment $E$ is obtained and the address of $P$ is assigned. Then, the first point of the contour of the burr is obtained from steps 2-5. Finally, the complete contour of the burr in the range of the window from $\left(x_{1}, y_{1}\right)$ to $\left(x_{2}, y_{2}\right)$ can be detected from steps $6-8$.

The advantages of BCTM are:

1. The grey level of the object need not be known a priori.

2. The noise of a line can be removed.

3. The connected problem of the contours of the burr in two adjacent images can be handled.

\subsection{Coordinate Transformation}

The disparity coordinates of the burr data must be transformed into the real coordinates for the practical control of the deburring process [19]. In order to save computation time, a linear relation is used between the disparity coordinates and the real coordinates. The linear coordinate transformation is

$$
\begin{aligned}
& x_{\mathrm{ri}}=a x_{\mathrm{d} i}+b y_{\mathrm{d} i}+c \\
& y_{\mathrm{r} i}=d x_{\mathrm{d} i}+e y_{\mathrm{d} i}+f
\end{aligned}
$$

where $\left[x_{\mathrm{ri}}, y_{\mathrm{ri}}\right]$ are the real coordinates of the $i$ th image pixel; $\left[x_{\mathrm{di}}, y_{\mathrm{d} i}\right]$ is the disparity coordinates of the $i$ th image pixel; and $a, b, c, d, e, f$ are the six parameters of the 2-dimensional transformation matrix. Since this transformation is linear, the transformation matrix

$$
\left[\begin{array}{lll}
a & b & c \\
d & e & f
\end{array}\right]
$$

can be easily solved from three pixel points and the computation takes only a short time. If the three pixel points are apart from one another and the angle between any two line segments formed by the three points is large enough, the precision of the coordinate transformation is good. In other words, the three points should not be collinear; otherwise, the inverse does not exist.

\subsection{Computation of the Data of the Burr}

The burr height of the real coordinates, $a_{\text {roor }}\left(x_{i}\right)$, is obtained by the BCTM algorithm and the coordinate transformation. The frequency of the burr $\left(f_{\text {burr }}\right)$ can be found by applying FFT to the $a_{\text {root }}\left(x_{i}\right)$, and the cross-section area of the burr $\left(A_{\text {burr }}\left(x_{i}\right)\right)$ can be solved from either equation (6) or equation (7).

By using the above algorithm, the required burr's data of $a_{\text {root }}\left(x_{i}\right), A_{\text {burr }}\left(x_{i}\right)$, and $f_{\text {burr }}$ can be obtained through the coordinate transformation and the FFT. The data can be incorporated with a suitable controller to perform accurate deburring tasks. 


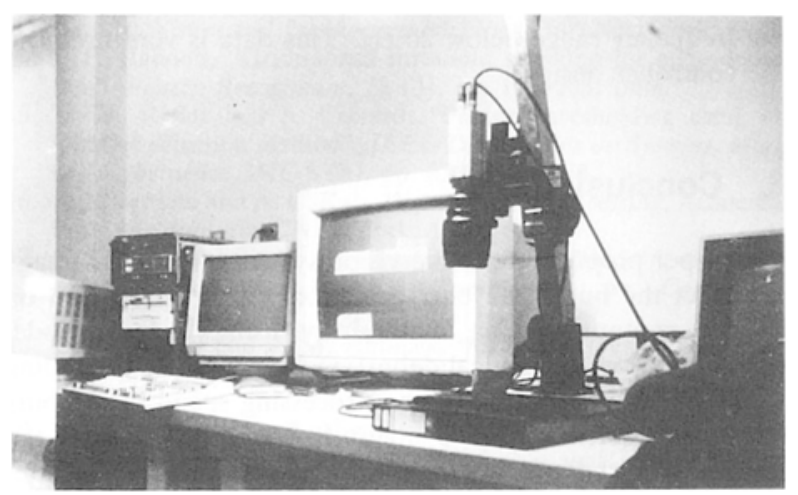

Fig. 6. The vision system for burr detection.

\section{Experiment}

The proposed image system uses a PC AT-386-25 equipped with an IM-1280 card, as shown in Fig. 6. The PC AT-386 does not have a coprocessor and runs on MS-DOS 3.3. The workpiece is an aluminium alloy. It is machined by a milling process, and the resultant burrs are rollover burrs (see Fig. 7). Hence, the parabolic burr model can be used to approximate the burrs. The burr length is about $66 \mathrm{~mm}$ and the burr radius is about $0.01 \mathrm{~mm}$.

The burr size on the image, as shown in Fig. 8, has been magnified by the magnifying lens to about 30 times the size of the original burr. Although each image picture can be represented by $480 \times 512$ pixels, only the $174 \times 360$ pixels in the centra area of the screen are used. This is due to the linearity requirement in the coordinate transformation. Since an image picture with $2 \mathrm{~mm}$ in height and $6 \mathrm{~mm}$ in width is represented by $174 \times 360$ pixels, the precision of the visual burr is about $0.016 \mathrm{~mm} /$ pixel. For each image frame, 360 pixels are processed by the BCTM for the burr contour. This process takes no more than $1 / 18.2 \mathrm{~s}$, i.e. the speed of the BCTM is $18.2 \mathrm{~Hz}$ per image frame.

The mesh diagram for the detected burr is shown in Fig. 9. The size and form is comparable to that of the actual burr. The burr height, the burr thickness and the cross-section area of the burr are shown in Figs. 10-12. These figures are similar

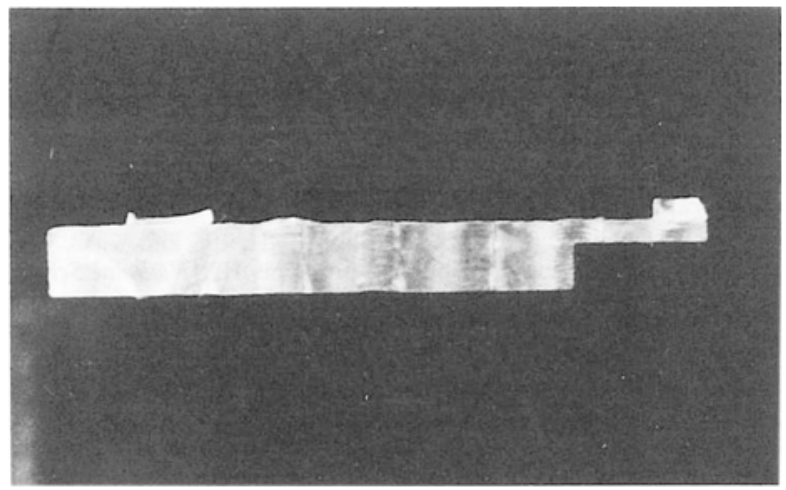

Fig. 7. The prototype of the rollover burr.

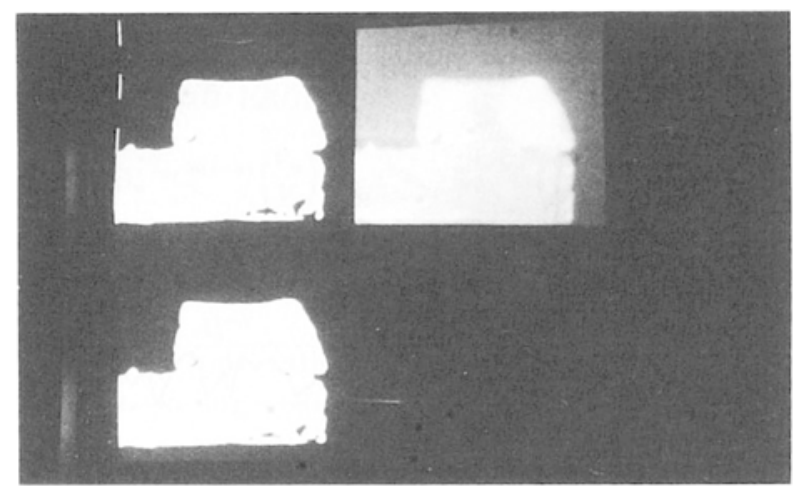

Fig. 8. The images of detected burrs.

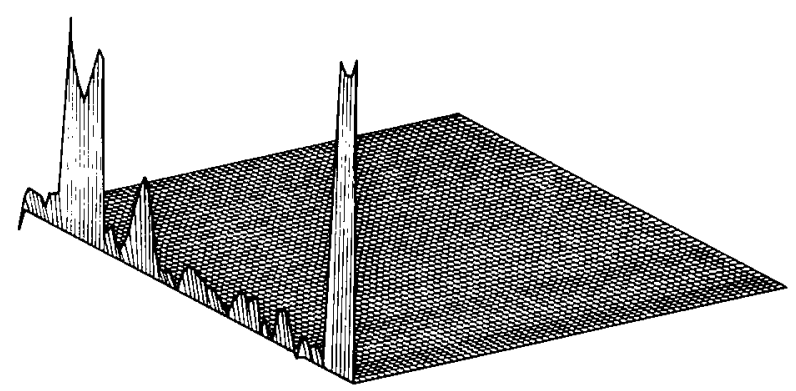

Fig. 9. The mesh diagram of the burr detected.

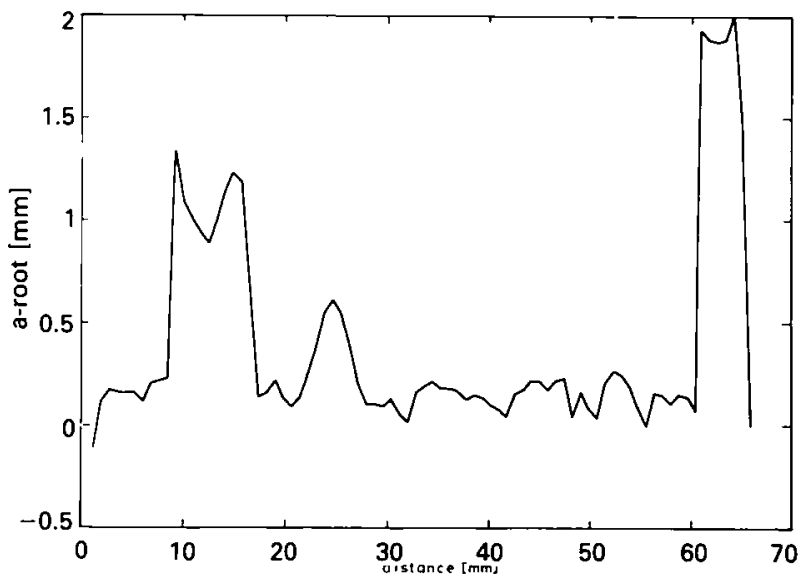

Fig. 10. The profile of the height of the burr detected.

in appearance, but their meanings and vertical scales are different. From these figures, we know that the maximum burr height, burr thickness, burr area, and burr length are $2.0 \mathrm{~mm}, 0.02 \mathrm{~mm}, 0.0267 \mathrm{~mm}^{2}$, and $66 \mathrm{~mm}$, respectively.

The range of the burr frequency will affect the control and the design of the end-effector for the deburring process. The DFT power spectrum of the burr with workpiece speed $V_{w}$ $=100 \mathrm{~mm} / \mathrm{s}$ is shown in Fig. 13. The power spectrum indicates the quantity of the burr which occurs at some fixed frequency. From Fig. 13, the main burr frequencies are located at 1.64, 


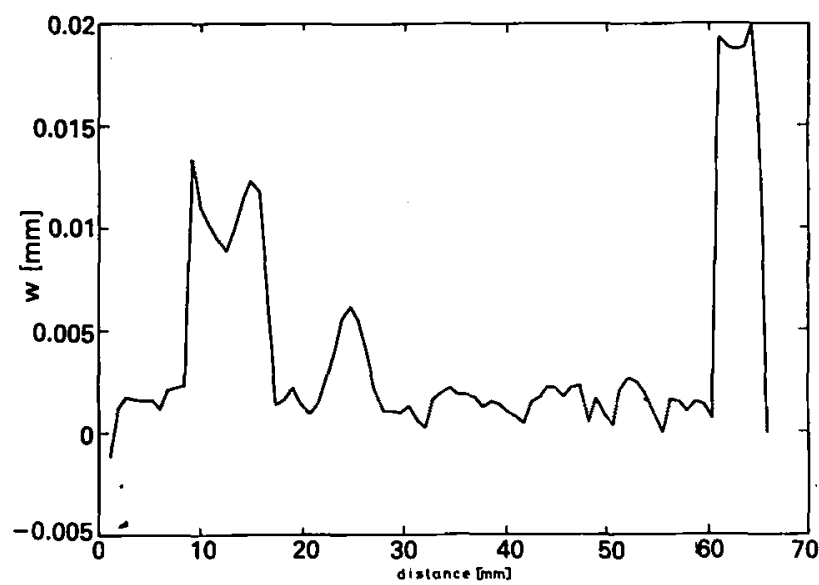

Fig. 11. The profile of the thickness of the burr detected.

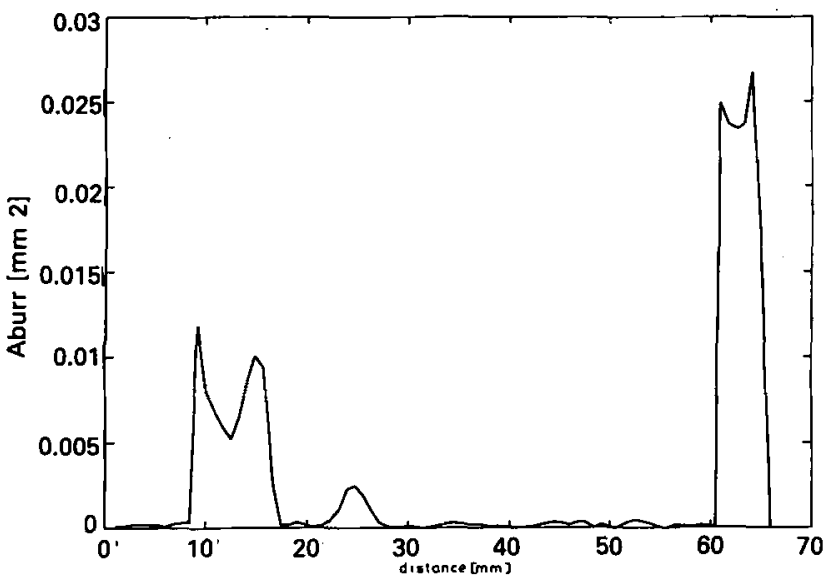

Fig. 12. The profile of the cross-section area of the burr detected.

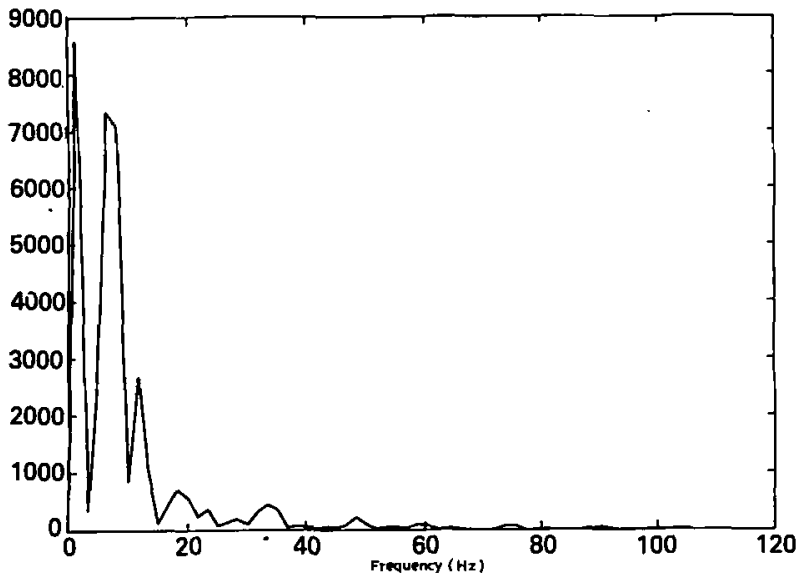

Fig. 13. The power spectrum of the burr detected.
$6.58,8.22,11.78$, and $18.36 \mathrm{~Hz}$, i.e. most of burrs cluster in the frequency range below $20 \mathrm{~Hz}$. This data is very useful in the controller design.

\section{Conclusion}

This paper proposes a passive vision system using a 2D image to detect the burr. The burr detection is primarily based on a burr contour-tracking method by using a burr model developed from a practical deburring force model. According to the experimental results, the processing speed of the burr detecting algorithm can be up to $18.2 \mathrm{~Hz}$. In addition, the precision of the measurement of this system is $0.016 \mathrm{~mm}$. This implies that the system is suitable for on-line burr detection and control. The experimental results show that the proposed vision system is an effective way to detect all data needed for the deburring processes. Though the experiment was performed only on the rollover burr, the system can be applied to all kinds of burrs. A robotic deburring system using this detection mechanism is under development.

\section{Acknowledgment}

Work on this paper is partially supported by the National Science Council, Republic of China under grant No. NSC790422-E-0022-03.

\section{References}

1. C. Rubenstein, "The mechanics of grinding", International Journal of Machine Tool Design and Research, 12, pp. 127-139, 1972.

2. R. S. Hahn and R. P. Lindsay, "Principles of grinding", Machinery Magazine, July-November 1971.

3. G. Boothroyd, Fundamentals of Metal Machining and Machine Tools, Scripta Book Company, 1975.

4. R. S. Hahn, "On the mechanics of the grinding process under plunge cut conditions", Journal of Engineering for Industry, February 1966.

5. H. Kazerooni, "Automated robotic deburring using electronic compliance: impedance control", Proceedings of IEEE International Conference of Robotics and Automation, pp. 1025-1032, 1987.

6. H. Kazerooni, J. J. Bausch and B. M. Kramer, "An approach to automated deburring by robot manipulators", ASME Journal of Dynamic Systems, Measurement, and Control, 108, pp. 354-359, 1986.

7. H. P. Huang, S. S. Lu and C. A. Shih, "Robotic deburring problems", Proceedings of the 5th CSME National Conference on Mechanical Engineering, pp. 769-779, 1988.

8. H. P. Huang, S. S. Lu and C. A. Shih, "Robot application to deburring problems I", National Science Council, NSC 77-0611E002-03, 1988.

9. H. P. Huang, S. S. Lu, C. A. Shih, M. H. Lin and K. C. Lee, "Robot application to deburring problems II", National Science Council, NSC 78-0422-E002-01, 1989.

10. H. P. Huang, S. S. Lu, C. A. Shih and M. H. Lin, "Robot application to deburring problems III", National Science Council, NSC 79-0422-E002-03, 1990.

11. Z. Aviad and E. Lozinskii, "Semantic thresholding", Pattern Recognition Letters, 5, pp. 321-328, 1987.

12. A. D. Brink, "Grey level thresholding of images using a correlation criterion", Pattern Recognition Letters, 9, pp. 335-341, 1989. 
13. K. S. Fu, R. C. Gonzales and C. S. G. Lee, Robotics: Control, Sensing, Vision and Intelligence, McGraw-Hill, New York, 1987.

14. J. F. Haddon, "Generalized threshold selection for edge detection", Pattern Recognition, 21 (3), pp. 195-203, 1988.

15. T. W. Ridler and S. Calvard, "Picture thresholding using an iterative selection method", IEEE Transactions on Systems, Man, and Cybernetics SMC-8 (8), pp. 630-632, 1978.

16. A. Rosenfeld and A. C. Kak, Digital Picture Processing, Academic Press Incorporation, New York, 1976.

17. J. S. Weszka, R. N. Nagel and A. Rosenfeld, "A threshold selection technique", IEEE Transactions on Computers, pp. 1322-1326, 1974.

18. O. A. Zuniga and R. M. Haralick, "Gradient threshold selection using the facet model", Pattern Recognition, 21 (5), pp. 493-503, 1988.

19. K. C. Lee and H. P. Huang, "Robot vision sensory assembly cell", Proceedings of the 7th CSME National Conference on Mechanical Engineering, pp. 1705-1712, 1990.

20. Y. Nakagawa and $A$. Rosenfeld, "A note on the use of local min and $\max$ operations in digital picture processing", $I E E E$ Transactions on Systems, Man, and Cybernetics SMC-8 (8), pp. $632-635,1978$.

21. G. Werner, "Influence of work material on grinding forces", Annals of the CIRP, 27 (1), pp. 243-248, 1978.

22. K. H. Fuh and J. S. Huang, "The study of force model for creep feed grinding", Proceedings of the 7th CSME National Conference on Mechanical Engineering, pp. 1583-1589, 1990.

23. F. Furukawa, S. Ohishi and S. Shiozaki, "Selection of creep feed grinding conditions in view of workpiece burning", Annals of the CIRP 28, 1, pp. 213-218, 1979.

24. C. E. Davis, "The dependence of grinding wheel performance of dressing procedure", International Journal of Machine Tool Design and Research, 14, pp. 33-52, 1974.

25. T. El-Wardani, M. M. Sadek and Y. Younis, "Theoretical analysis of grinding chatter", ASME Journal of Engineering for Industry, 109, pp. 314-320, November 1987.

26. H. Asada and N. Goldfine, "Optimal compliance design for grinding robot tool holders", Proceedings of IEEE International Conference of Robotics and Automation, pp. 316-322, March 1985.

27. J. J. Huang, "Top grinding technology", Mechanical Technology Magazine, Taipei, 1988.

28. S. Malkin, Grinding Technology, Ellis Horwood Limited, Chichester, England, 1989

29. Usuihideji, Technology of Cutting and Grinding, Japan, 1971.

30. D. E. Whitney, A. C. Edsall, A. B. Todtenkopt, T. R. Kurfess and A. R. Tate, "Development and control of an automated robotic weld bead grinding system", ASME Journal of Dynamic Systems, Measurement, and Control, 112, pp. 166-176, June 1990.

31. Y. Younis, M. M. Sadek and T. El-Wardani, "A new approach to development of a grinding force model", ASME Journal of Engineering for Industry, 109, pp. 306-313, November 1987.

\section{Appendix. Derivation of Force Models for Deburring Processes}

Werner [2I] proposed the following two functions to derive the force model for the grinding process:

$$
\begin{aligned}
& N_{\mathrm{d} y n}(l)=A_{n} C_{1}^{\beta}\left(\frac{V_{\mathrm{w}}}{V_{\mathrm{s}}}\right)^{\alpha}\left(\frac{a}{D}\right)^{q}\left(\frac{l}{l_{\mathrm{k}}}\right)^{\alpha} \\
& Q(l)=\frac{2}{A_{n}} C_{1}^{-\mathrm{\beta}}\left(\frac{V_{\mathrm{w}}}{V_{\mathrm{s}}}\right)^{1-\alpha}\left(\frac{a}{D}\right)^{1-\alpha}\left(\frac{l}{l_{\mathrm{k}}}\right)^{1-\alpha}
\end{aligned}
$$

where $N_{\text {dyn }}$ is the number of engaged cutting edges per wheel surface; $Q$ is the magnitude of the individual chip cross-section in the contact zone; $A_{n}$ is a proportional factor; $a$ is the cutting depth; $C_{1}$ is the static cutting edge density; $D$ is the equivalent wheel diameter; $l$ is the variable of the contact length; $l_{\mathrm{k}}$ is the contact length between the wheel and the workpiece; while $\alpha$ and $\beta$ are the exponential constants to describe the edge distribution: When $a \ll D$, the force model of the grinding process becomes

$$
\begin{aligned}
& F_{n}=K_{0} \int_{0}^{l_{k}} N_{\mathrm{dyn}}(l) \cdot[Q(l)]^{n} \mathrm{~d} l \\
& =2 K_{0} A_{n}^{1-n} C_{1}^{\mathrm{\beta}(1-n)}\left(\frac{V_{\mathrm{w}}}{V_{\mathrm{s}}}\right)^{2[\mid(1+n)+\alpha(1-n)\}^{2 \mid-1}}\left(\frac{a}{D}\right)^{[\alpha+n(1-\alpha)] / 2} \\
& \times l_{k}^{-\mid \alpha+n(1-\alpha)]} \int_{0}^{l} k \mid \alpha+n(1-\alpha) \mathrm{d} l \\
& =K_{0} \frac{A_{n}^{1-n}}{[1+\alpha+n(1-\alpha)] / 2} C_{1}^{\gamma}\left(\frac{V_{\mathrm{w}}}{V_{\mathrm{s}}}\right)^{2 \mathrm{e}-1}\left(\frac{a}{D}\right)^{[\alpha+n(1-\alpha))^{/ 2}} \\
& \times l_{k}^{-|\alpha+n(1-\alpha)|} l_{k}^{++n+a(1-n)} \\
& =K_{0} K C_{1}^{\gamma}\left(\frac{V_{w}}{V_{s}}\right)^{2 e-1}\left(\frac{a}{D}\right)^{e-1}(a D)^{\xi} \\
& =K_{0} K C_{1}^{\gamma}\left(\frac{V_{w}}{V_{s}}\right)^{2 e-1} a^{*} D^{1-*}
\end{aligned}
$$

where $l_{k}=\sqrt{\left[(D / 2)^{2}-(D / 2-a)^{2}\right]}=\sqrt{\left(a D-a^{2}\right)}=\sqrt{(a D)} ; \epsilon=$ $[(1+n)+\alpha(1-n)] / 2, \gamma=\beta(1-n) ; K=A_{n}^{1-n / \epsilon ;} K_{0}$ is the specific contact force per contact length; while $n$ is the exponential constant to describe the cutting process and $n=1$ for the pure chip formation process, but $n=0$ for the pure friction process.

However, equation (A3) should be modified when the cutting depth is significant compared to the wheel diameter [22]. In order to take into account the depth of cut, $N_{\mathrm{dyn}}$ and $Q$ in equations (A1) and (A2) should be modified so that they are functions of the contact angle. Namely,

$$
\begin{aligned}
& N_{\mathrm{dyn}}(\theta)=A_{n} C_{1}^{\beta}\left(\frac{V_{\mathrm{w}}}{V_{\mathrm{s}}}\right)^{\alpha}\left(\frac{a}{D}\right)^{\alpha / 2}\left(\frac{\sin \theta}{\sin \theta_{\mathrm{k}}}\right)^{\alpha} \\
& Q(\theta)=\frac{2}{A_{n}} C_{1}^{-\beta}\left(\frac{V_{\mathrm{w}}}{V_{\mathrm{s}}}\right)^{1-\alpha}\left(\frac{a}{D}\right)^{(1-\alpha) / 2}\left(\frac{\sin \theta}{\sin \theta_{\mathrm{k}}}\right)
\end{aligned}
$$

where $l=D \sin \theta / 2, l_{\mathrm{k}}=D \sin \theta_{\mathrm{k}} / 2$

Since the cutting force is the sum of the chip formation force and the friction force, and the specific normal chip formation force per active grain is $K_{1} Q$, the normal grinding force per active grain $\left(f_{\mathrm{n}}\right)$ and the tangential grinding force per active grain $\left(f_{1}\right)$ can be formulated as follows $[3,22-27 ; 1,6,28 ; 29-31]$

$$
\begin{aligned}
f_{\mathrm{n}}(\theta) & =\left(K_{1} Q(\theta)+\delta \bar{P}\right) N_{\mathrm{dyn}}(\theta) \\
f_{\mathrm{l}}(\theta) & =\left[\phi K_{1} Q(\theta)+\mu \delta \bar{P}\right] N_{\mathrm{dyn}}(\theta)
\end{aligned}
$$

where $K_{1}$ is the specific chip formation force per contact length; $\bar{P}$ is the average contact pressure; $\delta$ is the actual contact area between the wheel and the workpiece; $\delta \bar{P}$ is the specific normal friction force per active grain; $\mu$ is the ratio of the specific tangential friction force to the specific normal friction force; and $\phi$ is the ratio of the specific tangential chip formation force to the specific normal chip formation force. For the deburring process, $\alpha=0, \beta=\frac{1}{2}$. Substitute equations (A4) and (A5) into equations (A6) and (A7), we obtain

$$
\begin{aligned}
& f_{\mathrm{n}}(\theta)=2 K_{1}\left(\frac{V_{\mathrm{w}}}{V_{\mathrm{s}}}\right)\left(\frac{a}{D}\right)^{\dagger} \sin \theta / \sin \theta_{\mathrm{k}}+A_{n} C_{1}^{\dagger} \delta \bar{P} \\
& f_{\mathrm{l}}(\theta)=2 \phi K_{1}\left(\frac{V_{\mathrm{w}}}{V_{\mathrm{s}}}\right)\left(\frac{a}{D}\right)^{\dagger} \sin \theta / \sin \theta_{\mathrm{k}}+\mu A_{n} C_{1}^{\mathrm{j}} \delta \bar{P}
\end{aligned}
$$

For the up-cut grinding case (see Fig. A1), the vertical grinding force $F_{\mathrm{v}}$ and the horizontal force $F_{\mathrm{h}}$ can be obtained as

$$
\begin{aligned}
F_{\mathrm{v}}= & \int_{0}^{\theta_{k}}\left[f_{\mathrm{n}}(\theta) \cos \theta-f_{\mathrm{t}}(\theta) \sin \theta\right] \mathrm{d} \theta \\
= & K_{\mathrm{l}}\left(\frac{V_{\mathrm{w}}}{V_{\mathrm{s}}}\right)\left(\frac{a}{D}\right)^{\prime}\left[\sin \theta_{\mathrm{k}}-\phi\left(\theta_{\mathrm{k}} / \sin \theta_{\mathrm{k}}-\cos \theta_{\mathrm{k}}\right)\right] \\
& +\delta \bar{P} A_{n} C_{1}^{\ddagger}\left[\sin \theta_{\mathrm{k}}-\mu\left(1-\cos \theta_{\mathrm{k}}\right)\right]
\end{aligned}
$$




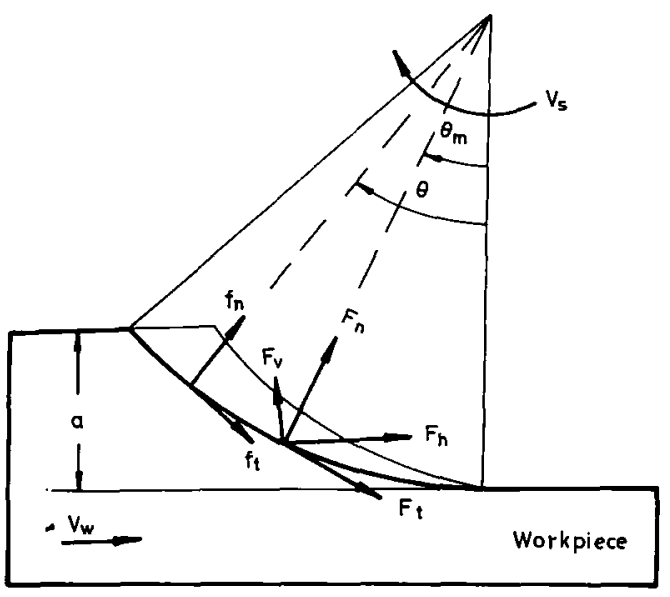

Fig. A1. The configuration of up-cut grinding.

$$
\begin{aligned}
F_{\mathrm{h}}= & \int_{0}^{\theta_{\mathrm{k}}}\left[f_{\mathrm{n}}(\theta) \sin \theta+f_{\mathrm{l}}(\theta) \cos \theta\right] \mathrm{d} \theta \\
= & K_{1}\left(\frac{V_{\mathrm{w}}}{V_{\mathrm{s}}}\right)\left(\frac{a}{D}\right)^{1}\left[\phi \sin \theta_{\mathrm{k}}+\left(\theta_{\mathrm{k}} / \sin \theta_{\mathrm{k}}-\cos \theta_{\mathrm{k}}\right)\right] \\
& +\delta \bar{P} A_{n} C_{\mathrm{j}}^{t}\left[\mu \sin \theta_{\mathrm{k}}+\left(1-\cos \theta_{\mathrm{k}}\right)\right]
\end{aligned}
$$

In addition, the normal grinding force $F_{\mathrm{n}}$ and the tangential grinding force $F_{\mathrm{t}}$ are obtained as follows

$$
\begin{aligned}
F_{\mathrm{r}}= & F_{\mathrm{v}} \cos \theta_{m}+F_{\mathrm{h}} \sin \theta_{\mathrm{m}} \\
= & K_{1}\left(\frac{V_{\mathrm{w}}}{V_{\mathrm{s}}}\right)\left(\frac{a}{D}\right)^{\prime}\left\{\left[\theta_{\mathrm{k}} \sin \theta_{\mathrm{m}} / \sin \theta_{\mathrm{k}}+\sin \left(\theta_{\mathrm{k}}-\theta_{\mathrm{m}}\right)\right]\right. \\
& \left.+\phi\left[\cos \left(\theta_{\mathrm{k}}-\theta_{\mathrm{m}}\right)-\theta_{\mathrm{k}} \cos \theta_{\mathrm{m}} / \sin \theta_{\mathrm{k}}\right]\right\} \\
& +\delta \bar{P} C_{\mathrm{l}}^{\dagger}\left\{\left[\sin \theta_{\mathrm{m}}+\sin \left(\theta_{\mathrm{k}}-\theta_{\mathrm{m}}\right)\right]\right. \\
& \left.+\mu\left[\cos \left(\theta_{\mathrm{k}}-\theta_{\mathrm{m}}\right)-\cos \theta_{\mathrm{m}}\right]\right\} \\
F_{\mathrm{t}}= & F_{\mathrm{h}} \cos \theta_{\mathrm{m}}-F_{\mathrm{v}} \sin \theta_{\mathrm{m}} \\
= & K_{1}\left(\frac{V_{\mathrm{w}}}{V_{\mathrm{s}}}\right)\left(\frac{a}{D}\right)^{\prime}\left\{\phi\left[\theta_{\mathrm{k}} \sin \theta_{\mathrm{m}} / \sin \theta_{\mathrm{k}}+\sin \left(\theta_{\mathrm{k}}-\theta_{\mathrm{m}}\right)\right]\right. \\
& \left.-\left[\cos \left(\theta_{\mathrm{k}}-\theta_{\mathrm{m}}\right)-\theta_{\mathrm{k}} \cos \theta_{\mathrm{m}} / \sin \theta_{\mathrm{k}}\right]\right\} \\
& +\delta \bar{P} A_{n} C_{1}^{\dagger}\left\{\mu\left[\sin \theta_{\mathrm{m}}+\sin \left(\theta_{\mathrm{k}}-\theta_{\mathrm{m}}\right)\right]\right. \\
& \left.-\left[\cos \left(\theta_{\mathrm{k}}-\theta_{\mathrm{m}}\right)-\cos \theta_{\mathrm{m}}\right]\right\}
\end{aligned}
$$

Similarly, we have the following equations for the down-cut grinding case (see Fig. A2),

$$
\begin{aligned}
F_{\mathrm{v}}= & \int_{0}^{\theta_{k}}\left(f_{n}(\theta) \cos \theta+f_{1}(\theta) \sin \theta\right) \mathrm{d} \theta \\
= & K_{1}\left(\frac{V_{\mathrm{w}}}{V_{\mathrm{g}}}\right)\left(\frac{a}{D}\right)^{\prime}\left[\sin \theta_{\mathrm{k}}+\phi\left(\theta_{\mathrm{k}} / \sin \theta_{\mathrm{k}}-\cos \theta_{\mathrm{k}}\right)\right] \\
& +\delta \bar{P}_{A_{n}} C_{\mathrm{i}}^{\mathrm{i}}\left[\sin \theta_{\mathrm{k}}+\mu\left(1-\cos \theta_{\mathrm{k}}\right)\right] \\
F_{\mathrm{h}}= & \int_{0}^{\theta_{k}}\left(-f_{\mathrm{n}}(\theta) \sin \theta+f_{\mathrm{k}}(\theta) \cos \theta\right) \mathrm{d} \theta \\
= & K_{1}\left(\frac{V_{\mathrm{w}}}{V_{\mathrm{k}}}\right)\left(\frac{a}{D}\right)^{\prime}\left[\mu \sin \theta_{\mathrm{k}}-\left(\theta_{\mathrm{k}} / \sin \theta_{\mathrm{k}}-\cos \theta_{\mathrm{k}}\right)\right] \\
& +\delta \bar{P}_{n} C_{1}^{\mathrm{l}}\left[\mu \sin \theta_{\mathrm{k}}+\left(1-\cos \theta_{\mathrm{k}}\right)\right]
\end{aligned}
$$

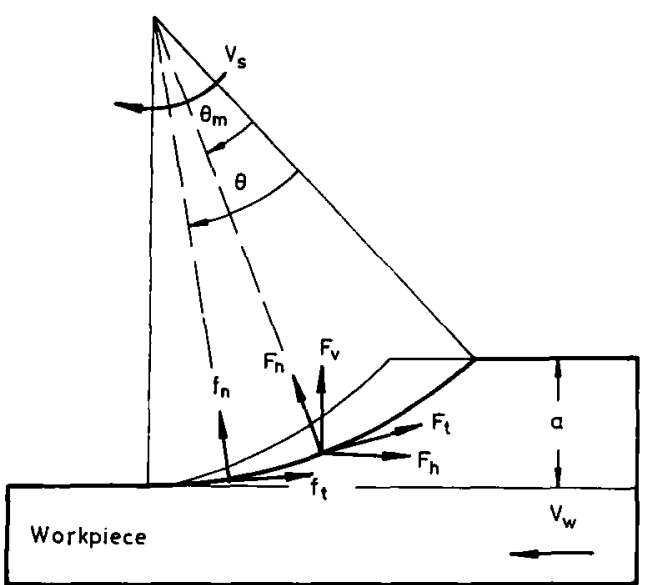

Fig. A2. The configuration of down-cut grinding.

$$
\begin{aligned}
& F_{\mathrm{n}}=F_{\mathrm{v}} \cos \theta_{\mathrm{m}}-\mathrm{F}_{\mathrm{h}} \sin \theta_{\mathrm{m}} \\
& =K_{1}\left(\frac{V_{\mathrm{w}}}{V_{\mathrm{s}}}\right)\left(\frac{a}{D}\right)^{\prime}\left\{\left[\theta_{\mathrm{k}} \sin \theta_{\mathrm{m}} / \sin \theta_{\mathrm{k}}+\sin \left(\theta_{\mathrm{k}}-\theta_{\mathrm{m}}\right)\right]\right. \\
& \left.-\phi\left[\cos \left(\theta_{k}-\theta_{m}\right)-\theta_{k} \cos \theta_{m} / \sin \theta_{k}\right]\right\} \\
& +\delta \bar{P} A_{n} C_{i}^{t}\left\{\left[\sin \theta_{m}+\sin \left(\theta_{k}-\theta_{m}\right)\right]\right. \\
& \left.-\mu\left[\cos \left(\theta_{k}-\theta_{m}\right)-\cos \theta_{m}\right]\right\} \\
& F_{\mathrm{t}}=F_{\mathrm{h}} \cos \theta_{\mathrm{m}}+F_{\mathrm{v}} \sin \theta_{\mathrm{m}} \\
& =K_{\mathrm{t}}\left(\frac{V_{\mathrm{w}}}{V_{\mathrm{s}}}\right)\left(\frac{a}{D}\right)^{t}\left\{\phi \left[\theta_{\mathrm{k}} \sin \theta_{\mathrm{m}} / \sin \theta_{\mathrm{k}}\right.\right. \\
& \left.\left.+\sin \left(\theta_{k}-\theta_{m}\right)\right]+\left[\cos \left(\theta_{k}-\theta_{m}\right)-\theta_{k} \cos \theta_{m} \sin \theta_{k}\right]\right\} \\
& +\delta \bar{P} A_{n} C_{\mathrm{i}}\left\{\mu\left[\sin \theta_{m}+\sin \left(\theta_{\mathrm{k}}-\theta_{\mathrm{m}}\right)\right]\right. \\
& \left.+\left[\cos \left(\theta_{k}-\theta_{m}\right)-\cos \theta_{m}\right]\right\}
\end{aligned}
$$

By examining equations ( $A 10)$ to ( $A 17$ ), we can see that the expressions for $F_{\mathrm{v}}, F_{\mathrm{h}}, F_{\mathrm{n}}$ and $F_{\mathrm{t}}$ are very complicated. From the experimental experience, the depth of cut $a$ is no larger than $1.5 \mathrm{~mm}$ and the equivalent diameter of the grinding wheel $D$ is about $20 \mathrm{~mm}$. In addition, $1-\cos \theta_{\mathrm{k}}=2 a / D \leq 0.15$. Therefore, $F_{\mathrm{v}}$ and $F_{\mathrm{h}}$ cannot be simplified. On the other hand, $F_{n}$ and $F_{1}$ can be further simplified.

Since the total cutting force is exerted at $\theta=\theta_{\mathrm{m}}$, the mean rotating angle $\theta_{\mathrm{m}} \simeq \theta_{\mathrm{k}} / 2$ from the geometrical relation in Fig. A3. In addition,

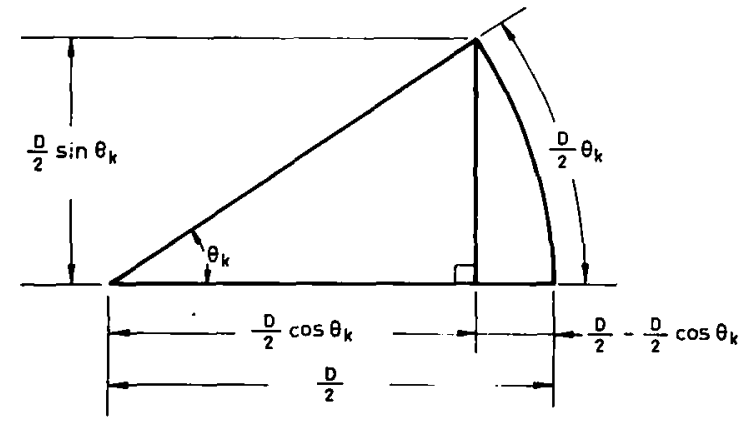

Fig. A3. The geometric relation of $\theta_{k}$. 


$$
\frac{1}{2} D \theta_{k}=\sqrt{\left\{\left(\frac{1}{2} D \sin \theta_{k}\right)^{2}+\left(\frac{1}{2} D-\frac{1}{2} D \cos \theta_{k}\right)^{2}\right\}}
$$

Dividing both sides of the above equation by $\frac{1}{2} D$, leads to

$$
\begin{aligned}
\theta_{k} & =\sqrt{\left[\sin ^{2} \theta_{k}+\left(1-\cos \theta_{k}\right)^{2}\right]} \\
& =\sqrt{\left(\sin \theta_{k}+1-2 \cos \theta_{k}+\cos ^{2} \theta_{k}\right)} \\
& =\sqrt{\left(2-2 \cos \theta_{k}\right)} \\
& =\sqrt{\left[2-2\left(\frac{1}{2} D-a\right) / \frac{1}{2} D\right]}
\end{aligned}
$$

Thus,

$$
\theta_{\mathrm{k}} \simeq 2\left(\frac{a}{D}\right)^{\prime}
$$

In addition,

$$
\cos \theta_{m} \simeq \cos \left(\theta_{k}-\theta_{m}\right)=\theta_{k} \cos \theta_{k} / \sin \theta_{k}
$$

and

$$
\begin{aligned}
& \theta_{k} \sin \theta_{m} / \sin \theta_{k}+\sin \left(\theta_{k}-\theta_{m}\right) \\
& \simeq 2 \theta_{m}=\theta_{k} \approx \sin \theta_{m}+\sin \left(\theta_{k}-\theta_{m}\right)
\end{aligned}
$$

Using the above relations in equations (A12), (A13), (A16) and (A17), we obtain the following equations for both the down-cutting and the up-cutting cases.

$$
\begin{aligned}
& F_{\mathrm{n}}=2 K_{1}\left(\frac{V_{\mathrm{w}}}{V_{\mathrm{v}}}\right) \frac{a}{D}+2 \delta \bar{P} A_{n} C_{1}^{4}\left(\frac{a}{D}\right)^{\prime} \\
& F_{\mathrm{t}}=2 \phi K_{1}\left(\frac{V_{\mathrm{w}}}{V_{\mathrm{s}}}\right) \frac{a}{D}+2 \mu \delta \bar{P} A_{n} C_{1}^{1}\left(\frac{a}{D}\right)^{\prime}
\end{aligned}
$$

In fact, the actual contact area $\delta$, the specific chip formation force per contact length $K_{1}$, and $\bar{P} A_{n} C_{1}^{4}$ can be further simplified. From equation (A18), the actual contact area $\delta$ can be derived as

$$
\delta=\frac{1}{2} D \theta_{k} L=\frac{1}{2} D 2(a / D)^{4} L=(a D)^{4} L
$$

where $L$ is the contact width between the wheel and the workpiece. Because the values of $K_{1}$ and the depth of cut $a$ depend on the cutting width $w$, the chip formation force should be modified as

$$
\int_{0}^{l} 2 K_{1}\left(\frac{V_{w}}{V_{\mathrm{s}}}\right) \frac{a(w)}{D} \mathrm{~d} w=\frac{2 K_{\mathrm{c}}}{D}\left(\frac{V_{\mathrm{w}}}{V_{\mathrm{s}}}\right) A_{\mathrm{work}}
$$

where $K_{\mathrm{c}}$ is the specific chip formation force per area; and $A_{\text {work }}$ is the cross-section area in the contact zone during deburring, shown in Fig. A4. Furthermore, we define the specific friction force per area, $K_{\mathrm{f}}$, as

$$
K_{1} \equiv \bar{P} A_{n} C_{1}^{1}
$$

Note that the depth of cut $a$ is equal to the burr height $a_{\text {roor }}$, shown in Fig. A5, in a deburring process. Substituting equations (A21) to (A23) into equations (A19) and (A20), we finally have

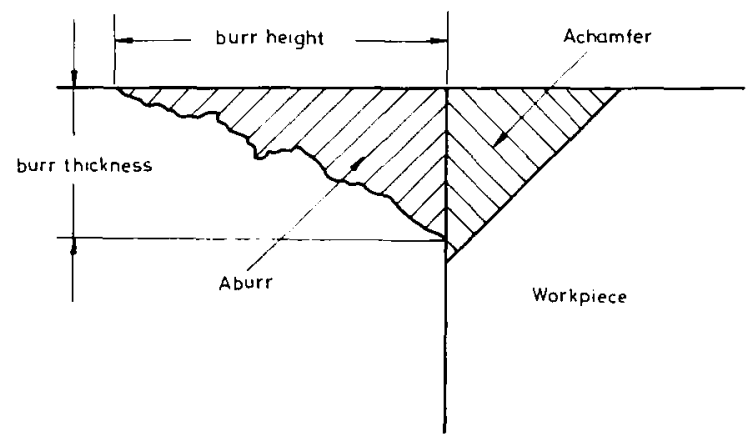

Fig. A4. The typical cross-section of the burr.

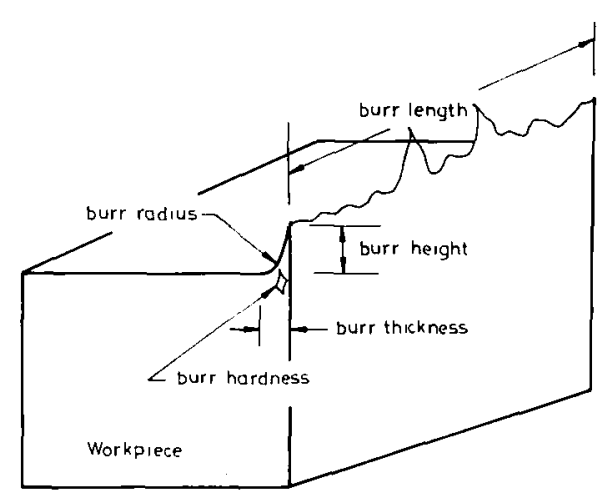

Fig. A5. The description of the burr data.

$$
\begin{aligned}
& F_{\mathrm{n}}=\frac{2 K_{\mathrm{c}}}{D}\left(\frac{V_{\mathrm{w}}}{V_{\mathrm{s}}}\right) A_{\text {work }}+2 K_{\mathrm{r}} a_{\text {roort }} L \\
& F_{\mathrm{t}}=\frac{2 \phi K_{\mathrm{c}}}{D}\left(\frac{V_{\mathrm{w}}}{V_{\mathrm{s}}}\right) A_{\text {work }}+2 \mu K_{\mathrm{r}} a_{\text {roort }} L
\end{aligned}
$$

Equations (A24) and (A25) denote the force model of the grinding process.

\section{Nomenclature}

$A_{\text {burr }} \quad$ cross-section area of the burr

$A_{\text {chamer }}$ cross-section area of the chamfer

$A_{n} \quad$ proportional factor

$A_{\text {work }} \quad$ cross section area in the contact zone while deburring

$A_{\text {work }}=A_{\text {burs }}+A_{\text {chamicr }}$

cutting width

$w$
$w_{\text {rexl }}$
$a$

thickness of the root of the burr

depth of cut

$\begin{array}{ll}a_{\text {rout }} & \text { burr height } \\ & a_{\text {roort }}=a\left(w_{\text {roxot }}\right)\end{array}$

$C_{1} \quad$ static cutting edge density

$D$ equivalent wheel diameter

$d_{\mathrm{s}} \quad$ wheel diameter

$d_{\mathrm{w}} \quad$ workpiece diameter

$D=d_{w} d_{\mathrm{s}} /\left(d_{\mathrm{w}} \pm d_{\mathrm{s}}\right)$

$D=d_{\mathrm{s}}$ and $d_{w} \rightarrow \infty$ for the deburring process

$F_{\mathrm{h}} \quad$ horizontal grinding force

$F_{\text {v }} \quad$ vertical grinding force

$F_{n} \quad$ normal grinding force

$F_{1} \quad$ tangential grinding force

$F_{\text {n(K) }}$

$F_{\text {l(K) }}$

$F_{0}$

normal grinding force of the Kazerooni's model

tangential grinding force of the Kazerooni's model

threshold thrust force

burr frequency

normal grinding force per active grain

tangential grinding force per active grain

first resonant frequency of the robot

resonant frequency of the end-effector at the normal direction

exponential constant for describing the edge distribution $\epsilon=[(1+n)+\alpha(1-n)] / 2$

$\epsilon=(1+n) / 2$ for $\alpha=0[21]$

$K$ proportional factor of the force model of the grinding process

$K=A_{n}^{b-n} / \epsilon$ 
$K_{0} \quad$ specific contact force per contact length

$K_{1} \quad$ specific chip formation force per contact length

$V_{s} \quad$ wheel speed

$V_{w} \quad$ workpiece speed

$\wedge_{w} \quad$ metal-removal parameter

$K_{2} \quad$ specific metal-removal parameter per wheel speed $K_{2}=\wedge_{\mathrm{w}} / V_{\mathrm{s}}$

$K_{\mathrm{c}} \quad$ specific chip formation force per area

$K_{\mathrm{f}} \quad$ specific friction force per area

$k$ constant for the parabolic burr

$k_{1}, k_{2}, k_{3}, k_{4}$ constants for the circular burr

$L \quad$ contact width between the wheel and the workpiece

$L$ is equal to the chamfer's hypotenuse length, or $L=$ $w_{\text {root }}$ when there is no chamfer

$l_{k} \quad$ contact length

$\begin{array}{ll}l_{k} & \text { contact length between the wheel and the workpiece } \\ m & \text { exponential constant for describing the edge shape }\end{array}$

$$
0 \leq m \leq 1
$$

$m=1$ for the deburring process [21]

$N_{\text {dyn }} \quad$ number of engaged cutting edges per wheel surface

$n$ exponential constant for describing the cutting process $0 \leq n \leq 1$

$n=1$ for the pure chip formation process and $n=0$ for the pure friction process [22]

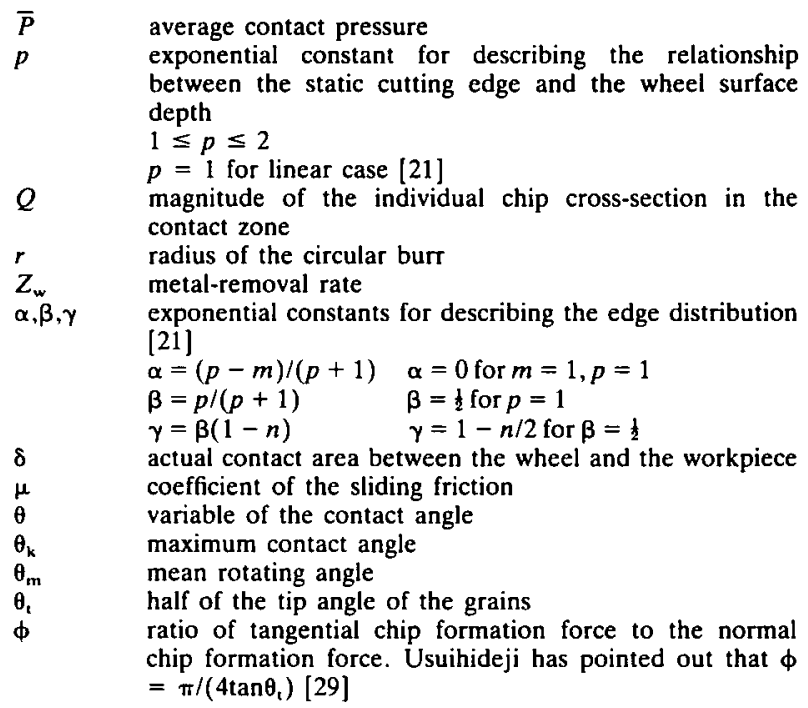
between depth$$
1 \leq p \leq 2
$$

magnitude of the individual chip cross-section in the contact zone

$r \quad$ radius of the circular bur

$Z_{w} \quad$ metal-removal rate [21]$$
\gamma=\beta(1-n)
$$$$
\beta=\frac{1}{2} \text { for } p=1
$$
actual contact area between the wheel and the workpiece coefficient of the sliding friction
maximum contact angle $=\pi /\left(4 \tan \theta_{1}\right)[29]$

